Meta

Journal des traducteurs

Translators' Journal

\title{
La synonymie en langue de spécialité
}

\section{Jacques Lethuillier}

Volume 34, numéro 3, septembre 1989

1. Actes du Colloque Les terminologies spécialisées : Approches quantitative et logico-sémantique et 2 . Actes du Colloque Terminologie et Industries de la langue

URI : https://id.erudit.org/iderudit/003498ar

DOI : https://doi.org/10.7202/003498ar

Aller au sommaire du numéro

Éditeur(s)

Les Presses de l'Université de Montréal

ISSN

0026-0452 (imprimé)

1492-1421 (numérique)

Découvrir la revue

Citer cet article

Lethuillier, J. (1989). La synonymie en langue de spécialité. Meta, 34(3),

443-449. https://doi.org/10.7202/003498ar d'utilisation que vous pouvez consulter en ligne.

https://apropos.erudit.org/fr/usagers/politique-dutilisation/ 


\section{LA SYNONYMIE EN LANGUE DE SPÉCIALITÉ}

JACQUES LETHUILLER

Université de Montréal, Montréal, Canada

Invité à une émission récente de Bernard Pivot, APOSTROPHES, M. Laplante, grand directeur de la traduction de l'œuvre de Freud, expliquait qu'il n'y a pas de véritable synonymie.

Pour le Robert, les synonymes sont des mots ou expressions qui ont le même sens ou une signification très voisine. Dubois parle de synonymie complète et de synonymie incomplète. Le concept de synonymie complète est lié à la distinction que l'on fait entre le sens cognitif et le sens affectif. Cette distinction, si elle est applicable aux mots de la langue courante, ne peut être étendue facilement aux TERMES des langues de spécialité.

En langue de spécialité, la synonymie vraie existe. Elle s'inscrit dans le processus de désignation des notions, autrement dit, de désignation des classes d'objets particuliers dites terminologiques.

Il ne faut pas confondre synonymie et interchangeabilité en discours de formes linguistiques concurrentes. Pour nous, des termes sont SYNONYMES dès lors que leur fonction première, celle de DÉSIGNATION ou de POINTEUR DE CLASSE D'OBJETS PARTICULIERS, est la même. Cela n'empêche pas qu'ils aient des attributs secondaires (motivation, longueur, qualité linguistique, facilité de mémorisation, etc.) différents et que, par conséquent, leur emploi soit plus adapté dans un type de discours que dans un autre.

Expliquer la synonymie, c'est d'abord essayer de faire comprendre la fonction de désignation de la terminologie. On ne peut cependant parler de cet aspect sans rappeler brièvement le fonctionnement de la terminologie.

Les systèmes terminologiques sont des systèmes de classement et de description des objets particuliers - abstraits ou concrets. Ils groupent les objets particuliers en ensembles appelés CLASSES, que servent à pointer des formes linguistiques appelées TERMES, selon leur conformité à des schémas de description dits NOTIONS. Il y a synonymie lorsque plusieurs formes linguistiques ou termes servent à désigner ou POINTER concurremment UNE MÊME CLASSE. Ce phénomène correspond à une certaine redondance de la langue, dans laquelle on peut voir une richesse ou au contraire des risques de confusion.

À ce point, il convient de s'interroger sur la nature précise de ce que servent à évoquer ces formes linguistiques appelées termes et sur la nature des critères employés pour les choisir ou les élaborer. On a parlé de classes ou ensembles d'objets particuliers. On aurait pu dire de la même façon que ces termes servent à pointer vers les schémas de description ou notions qui sont associés à ces ensembles privilégiés par la terminologie et les délimitent. C'est, dans le langage courant, le SENS attaché aux termes.

Le choix ou la formation des termes jouant le rôle d'étiquettes ou de pointeurs collectifs pour des classes d'objets particuliers présente une part d'arbitraire, de convention, mais il n'en demeure pas moins que le terme, pour remplir le plus adéquatement sa fonction de désignation, doit évoquer le mieux possible le schéma de description ou NOTION associé à la classe, le schéma qui est commun à tous les objets de la classe. 
Cela nous renvoie encore à l'idée de schéma ou de notion, qui est difficile à cerner parce que sa définition est entachée de circularité.

Une NOTION est un agrégat de caractères. Et un CARACTÈRE est une relation entre des objets appartenant à des classes différentes, donc conformes à des schémas de description ayant le plus souvent peu de caractères en commun. Par conséquent, une NOTION est un ensemble de relations (permanentes), toujours vraies, qu'entretiennent les objets d'une classe avec ceux d'autres classes.

Revenons à la démarche de désignation des classes terminologiques par des formes linguistiques. Grâce au TERME POINTEUR, on doit évoquer à la fois : CLAIREMENT (sans ambiguité, notamment) et ÉCONOMIQUEMENT (avec concision) la notion ou schéma commun à tous les objets d'une classe. Dès qu'un schéma devient riche, c'est-àdire qu'il correspond à une classe restreinte, il est impossible, au moyen d'une forme linguistique répondant à de tels critères, de lister tous les caractères (toutes les relations significatives et permanentes avec des objets d'autres classes) qui forment le schéma notionnel.

Heureusement, les systèmes terminologiques utilisent le principe de l'héritage. Leur structure est arborescente. Toute classe se rattache à une classe plus large, qui correspond à un schéma moins riche, donc à une notion plus générale. Dans ces conditions, la manière la plus simple de désigner une classe particulière consiste à s'appuyer sur le pointeur de la classe plus large la plus petite dont elle découle, le genre le plus proche, et à le spécifier en évoquant la ou les valeurs du ou des caractères sur laquelle ou lesquelles se fonde la classe considérée (un seul caractère dans le cas le plus simple). On aboutit au schéma de base suivant:

$\begin{array}{cll}\text { TERME SPÉCIFIQUE }=\quad \begin{array}{l}\text { GÉNÉRIQUE } \\ \text { DÉTERMINÉ } \\ \text { hyperonyme } \\ \text { noyau } \\ \text { syntagmatique } \\ \text { co-hyponyme }= \\ \text { SPÉCIFIQUE }=\end{array} & \begin{array}{l}\text { DÉTERMINANT } \\ \text { évocation de la } \\ \text { valeur du caractère } \\ \text { pertinent }\end{array} \\ & \text { nouveau noyau syntagmatique }\end{array}$

qui est celui de la DÉNOMINATION SYNTAGMATIQUE.

Le processus de dénomination syntagmatique est récurrent. Un pointeur de classe spécifique peut à son tour jouer le rôle de noyau syntagmatique dans la désignation de sous-classe encore plus spécifiques, et ainsi de suite.

La longueur de la forme linguistique utilisée comme POINTEUR devient rapidemment inacceptable.

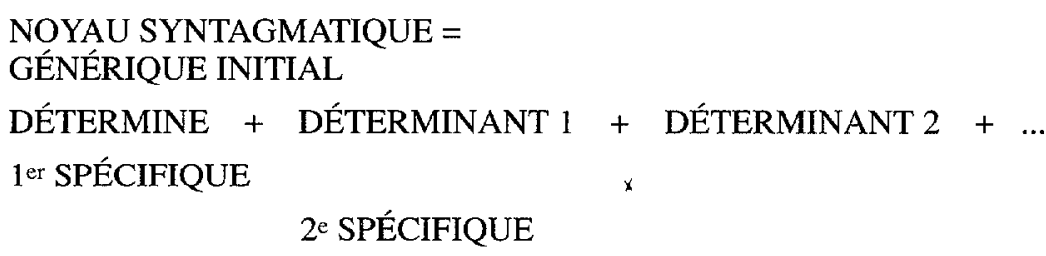

On peut avoir couramment, par exemple, cinq expansions correspondant chacune à une particularisation par adjonction d'un caractère au schéma de description correspon- 
dant au noyau syntagmatique, c'est-à-dire au générique de départ dont le POINTEUR est toujours conservé.

Pour chacune des composantes, des ressources linguistiques concurrentes, porteuses du même sens, sont utilisables. Ces formes concurrentes peuvent appartenir à des fonds différents ou exploiter la métaphore pour exprimer la valeur d'un caractère. Par combinaison, cela peut donner naissance à un nombre élevé de variantes synonymiques, en particulier s'il y a plusieurs composantes et que chacune d'elles soit exprimable de plusieurs façons.

\title{
HÉLICE À PAS VARIABLE À CALAGE DES PÂLES VARIABLE \\ HÉLICE AUX PROFILS AUTORÉGULANTS \\ HÉLICE À RÉGLAGE AUTOMATIQUE HÉLICE À PAS VARIABLE AUTOMATIQUE
}

Les variantes ainsi formées ont souvent des fréquences d'emploi comparables et ne se démarquent pas les unes des autres dans l'usage.

La désignation syntagmatique par expansions multiples successives a ses limites. Après deux ou trois particularisations successives avec conservation du noyau syntagmatique, le terme deviendrait inacceptable si des formes réduites n'étaient pas utilisables concurremment.

Les procédés de réduction radicaux, avec suppression du noyau syntagmatique, surmontent la difficulté de façon très efficace en ramenant le syntagme dénominatif à une forme monolithique.

\author{
MOTEUR ÉOLIEN \\ ÉOLIENNE \\ AÉROMOTEUR \\ MACHINE MOTRICE \\ MOTEUR \\ POMPE À CHALEUR \\ THERMOPOMPE \\ RADAR DOME \\ RADOME \\ RAM \\ RANDOM ACCESS MEMORY \\ MEV \\ MÉMOIRE VIVE
}

À côté de ces procédés, qui comprennent la siglaison (laser, maser, radar, sida, RAM, MEV, EPROM, ROM, etc.) et différents types de fusion (apocope, aphérèse, syncope) bien connus et dont l'effet sur la motivation des termes est très franc (celle-ci devient pratiquement nulle), il existe un procédé «doux», très largement utilisé pour rendre acceptables les désignations syntagmatiques très spécifiques. C'est l'ellipse, qui conserve le noyau syntagmatique, contrairement aux procédés de réduction ci-dessus.

L'ellipse conduit à des synonymes moins opaques en apparence que la siglaison ou la réduction à une forme monolithique pure et simple par fusion. Néanmoins, du fait qu'elle gomme une série de caractères fondant des classes spécifiques intermédiaires, son 
effet sur la motivation des termes est pernicieux. Les termes deviennent faussement transparents.

Ce type de réduction est hypocrite. Il nous laisse croire à un rapport direct entre le déterminé et le déterminant, alors que ce dernier peut se trouver à «une bonne distance» (en nombre de classes spécifiques intermédiaires) de la classe générique représentée par le noyau syntagmatique.

Qu'est-ce qu'un «moteur culbuté»? Tout d'abord, ce terme, je devrais dire «culbuté» ne s'applique qu'aux moteurs (thermiques) (à combustion interne) (volumétrique) (à pistons alternatifs). Et le déterminant ne s'applique en fait qu'à un type particulier de composant, la ou les soupape(s). Un moteur (à pistons) culbuté est un moteur (à soupapes) culbutées, c'est-à-dire à soupapes dont le mouvement de levée se fait par l'intermédiaire d'un culbuteur ou basculeur qui renvoie vers le bas le déplacement de bas en haut de la tige actionnée par une came de l'arbre à cames.

De la même façon, on peut se demander ce qu'est un moteur «carré». Encore ici, le déterminant «carré» caractérise un moteur à pistons et, plus précisément, un moteur (thermique) (à combustion interne) (volumétrique) à pistons alternatifs. On pourrait croire purement et simplement qu'un moteur carré désigne un moteur à pistons de forme carrée. Il n'en est rien et cette unité est bien un terme. Un moteur carré est un moteur à rapport course/alésage égal à l'unité. La désignation est en quelque sorte métaphorique ici, puisque c'est la coupe du volume balayé par le piston qui possède une forme carrée.

rapport course/

alésage

\section{MOTEUR À PISTON}

$<1$

Moteur plat

$$
=1
$$

Moteur carré
$>1$

Moteur à longue course

Notons que les réductions par ellipse ont des inconvénients au chapitre de l'apprentissage, dans la mesure où lorsque des termes présentent une ressemblance quant à la forme, on a tendance à vouloir les interpréter de la même façon.

Prenons le cas des deux couples suivants:

TURBINE À GAZ

TURBINE À VAPEUR

\section{MOTEUR À GAZ \\ MOTEUR À ESSENCE}

Ces deux couples de termes sont intéressants à examiner.«Turbine à gaz» s'oppose à «turbine à vapeur». La même ellipse y est faite. Il faudrait interpréter ces termes comme: «(moteur) turbine à (fluide moteur de type) gaz» et «(moteur) turbine à (fluide moteur de type) vapeur».

L'ellipse est différente pour le couple suivant. Il s'agit cette fois de «moteur à (combustible du type) essence» et de «moteur à (combustible du type) gaz».

Dans chacun des couples, les objets de la grande classe générique sont en RAPPORT TOUT-PARTIE avec :

«fluide moteur», et avec

«combustible». 
Il s'agit de caractères impliqués hérités en fait, qui remontent à «moteur thermique» pour «fluide moteur» et à «moteur thermique à combustion» pour combustible.

La désignation syntagmatique est un moyen terme entre la désignation périphrastique toujours très fluctuante et la réduction à une FORME MONOLITHIQUE par FUSION (apocope, aphérèse, syncope) ou par SIGLAISON, toujours très stable. Tous les éléments de sens des expansions successives, ajoutés à ceux du noyau syntagmatique, sont reportés dans une nouvelle forme à partir de laquelle reprendra la dénomination syntagamatique; on a «remis à zéro le compteur».

Cette réduction radicale est à l'origine de synonymies.

MOTEUR ÉOLIEN
ÉOLIENNE
AÉROMOTEUR
POMPE À CHALEUR
THERMOPOMPE

Il s'agit toujours d'une synonymie de forme dans la mesure où les aspects évoqués plus ou moins implicitement par les formes ainsi condensées radicalement demeurent les mêmes que les aspects évoqués par les DÉNOMINATIONS SYNTAGMATIQUES dont elles découlent.

Ce n'est pas cette synonymie de forme, très bien traitée déjà, même si nombre d'études n'examinent que rarement les rapports entre formes linguistiques et notions, qui a retenu le plus notre attention.

$\grave{A}$ côté de cette synonymie de forme, il existe une synonymie de FOND, encore qualifiée de synonymie d'ASPECT par certains, d'autant plus intéressante qu'elle est liée à un aspect fondamental du système de NOTIONS et non plus seulement au système des FORMES LINGUISTIQUES.

Dans la synonymie de FOND, les formes linguistiques concurrentes pointent toujours vers une même classe d'objets particuliers, mais évoquent des caractères différents de la NOTION. Comment est-ce possible sans que les systèmes perdent leur cohérence?

Rappelons une fois encore qu'une NOTION n'est pas autre chose qu'un SCHÉMA DE DESCRIPTION, sous la forme d'un agrégat de caractères, et, pour être plus précis, d'une série de RELATIONS (primitives de sens) entretenues par les objets conformes à ce schéma avec des objets conformes à d'autres schémas ou NOTIONS.

Ajoutons qu'il est utile, voire essentiel, de distinguer le schéma RÉDUIT, formé de tous les CARACTÈRES PERTINENTS hérités, qui fondent les classes génériques successives auxquelles se rattache la classe considérée, du schéma COMPLET qui réunit tous les caractères impliqués ou CONSÉCUTIFS, à tous les niveaux.

Le premier schéma est en fait le squelette de la notion, sa SIGNATURE. Il représente l'ENSEMBLE DE TRAITS MINIMAL en fixant la place dans le réseau notionnel, sans ambiguïté.

Ce squelette est enrobé d'une SUBSTANCE qui constitue les caractères dits CONSÉCUTIFS ou caractères IMPLIQUÉS, AUTOMATIQUES. Ce sont CES traits impliqués qui forment la base des descriptions encyclopédiques RACCROCHÉES aux définitions terminologiques exprimant l'ensemble des traits minimaux selon un schéma formel. Seuls, d'ailleurs, les traits impliqués nouveaux, ceux qui découlent des traits pertinents FONDANT le SPÉCIFIQUE, rentrent en principe dans le schéma encyclopédique. Ils apparaissent comme une liste de relations à caractère permanent, TOUJOURS VRAIES. Ils sont en outre utilisables EN AVAL comme palette de description, comme nouvelle INDÉTERMINATION représentant une série de virtualités, une série de possi- 
bilités de réalisation susceptible de créer des oppositions à partir desquelles des sousclasses encore plus spécifiques pourront être FONDÉES. Ils sont donc utilisables comme caractères pertinents EN AVAL de la NOTION dont ils constituent la SUBSTANCE.

La question se pose de savoir à quelle condition ces différents caractères consécutifs peuvent être utilisés pour former des synonymes qui seront des DOUBLETS DE FOND.

Il faut que le caractère consécutif soit réalisé dans la seule sous-classe considérée, qu'il ne s'applique pas à d'autres objets de la grande classe de rattachement (genre le plus proche). Sinon le système serait menacé d'incohérence.

Si cette condition est satisfaite, on peut parler de correspondance biunivoque entre la valeur du caractère pertinent ayant fondé la sous-classe et le caractère consécutif considéré, cela dans l'espace conceptuel limité par la NOTION GÉNÉRIQUE.

On pourrait se demander pourquoi attacher autant d'importance à un phénomène qui semble limité quantitativement. Il y a plusieurs raisons à cela, outre le fait que ce phénomène a suscité peu d'intérêt jusqu'à présent chez les terminologues.

La première raison peut-être, c'est que jeter une certaine lumière sur cet aspect de la désignation peut aider à faire comprendre certains aspects du fonctionnement de la terminologie.

Par ailleurs, dans l'enseignement des langues de spécialité, l'existence de variantes synonymiques de fond plus fortement motivées que les termes formés à partir du noyau syntagmatique et d'un déterminant qui évoque le caractère pertinent (sur lequel est fondée la notion) nous paraît révéler une richesse linguistique dont il importe d'être conscient et qui mérite d'être exploitée.

Le terminologue et le traducteur, en outre, sont directement intéressés par cette forme de synonymie quand elle devient interlinguistique. Et il convient de noter que si la SYNONYMIE DE FOND unilingue n'est pas très répandue, la SYNONYMIE DE FOND interlinguistique est abondante.

$$
\begin{array}{ll}
\text { SLIDING RULE } & =\text { RÈGLE À CALCUL } \\
\text { SCREEN } & =\text { MOUSTIQUAIRE } \\
\text { TUNING FORK } & =\text { DIAPASON }
\end{array}
$$

Ce dernier point soulève la question toujours très épineuse de la reconnaissance du TERME en discours. Un groupe de mots sémantiquement monolithique doit-il être pris pour un TERME ou pour une UNITÉ DE DISCOURS ? Cette question est aussi critique pour le traducteur que pour le terminologue. La synonymie de fond, tant unilingue qu'interlinguistique, constitue un critère de reconnaissance du terme que l'on n'a pas souvent mis de l'avant. Au même titre que la présence du TERME dans un dictionnaire, il s'agit d'une CONDITION SUFFISANTE mais NON NÉCESSAIRE pour qu'une UNITÉ repérée en discours puisse être considérée comme un TERME.

La possibilité d'utiliser la SYNONYMIE DE FOND comme critère de reconnaisance du terme (quand celui-ci est du type syntagmatique), découle en fait d'un autre critère, plus général, en rapport direct avec l'idée de NOTION, qui, d'ailleurs, n'est pas davantage cité dans les écrits sur la terminologie. Il s'agit cette fois d'une CONDITION NÉCESSAIRE ET SUFFISANTE.

Pour qu'une unité ayant la forme suivante:

$$
\text { DÉTERMINÉ + DÉTERMINANT }
$$

représente un terme, il faut et il suffit que l'équation ci-après soit vérifiée :

Sens (DÉTERMINÉ + DÉTERMINANT) > Sens (DÉTERMINÉ) + Sens (DÉTERMINANT). 

vante:

Ce qui se cache derrière cette formule en apparence fantaisiste, c'est l'équation sui-

$$
\text { Sens (DÉTERMINÉ + DÉTERMINANT) }
$$

pointeur de la

classe spécifique

notion spécifique

Sens (DÉTERMINÉ) + Sens (DÉTERMINANT) + [Caractères consécutifs]

$\begin{array}{ll}\text { pointeur de } & \text { valeur du } \\ \text { la classe } & \text { caractère } \\ \text { générique } & \text { pertinent }\end{array}$

notion générique

notion spécifique

C'est le fait que l'eau lourde est plus que de l'eau lourde, par exemple.

Le fait qu'un moteur thermique ou moteur à énergie thermique est plus qu'une machine motrice qui transforme en travail de la chaleur, que les objets de cette classe «moteur thermique» possèdent d'autres caractères permanents qui, s'ils sont EXCLUSIFS, peuvent servir à désigner la classe sans ambiguïté.

Il y a un DÉCROCHAGE entre le SENS ADDITIONNÉ et le SENS GLOBAL. Et l'on peut poser en HYPOTHÈSE que c'est l'importance de cet écart qui mesure le DEGRÉ DE LEXICALISATION d'un terme, dont on parle tant dans les textes de façon généralement abstraite.

Ce critère rejoint celui d'opacité de Rey. Un exemple fera sans doute mieux comprendre notre propos...(planche sur moteur d'avion, moteur d'automobile, etc.).

Le rapport avec la SYNONYMIE DE FOND? Si une forme linguistique composée est employée par un auteur, CONCURREMMENT en apparence avec une autre forme dont la partie DÉTERMINANT semble évoquer quelque chose de radicalement différent, on a de fortes raisons de croire que les formes rencontrées sont des TERMES.

Pour être plus explicite, si un discours utilise des SYNONYMES DE FORME, de type syntagmatique (non rendus opaques par siglaison ou par d'autres procédés de réduction visibles), et cela de façon concurrente, pour évoquer le même sens d'après le contexte, on peut toujours croire que l'interprétation doit être celle des sens ADDITIONNÉS des composants, autrement dit, qu'il ne s'agit pas nécessairement de termes.

Dans le cas d'une situation identique avec des SYNONYMES DE FOND, donc dont les déterminants évoquent des choses radicalement différentes, la présomption est forte qu'il s'agisse de TERMES. 